plasma D-dimer level was low $\left(282 \mu \mathrm{g} \cdot \mathrm{L}^{-1}\right)$. Chest radiography showed multiple ill-defined increased densities in both lower lung fields. High-resolution computed tomography was then performed and showed ill-defined, wedge-shaped increased densities with patent airways in the posterior subpleural regions of both lower lobes. The computed tomography differential diagnosis was organising pneumonia, bronchopneumonia, Churg-Strauss syndrome, Wegener granulomatosis, pulmonary haemorrhage, or vasculitis of various causes. Transbronchial lung biopsy was performed in the right lower lobe. Microscopic examination showed alveolar haemorrhage without evidence of vasculitis, eosinophilic infiltration or organising pneumonia. Serum circulating antiphospholipid antibodies were eventually found. Therefore, pulmonary angiography was performed and an intra-arterial low density was found in the right main pulmonary artery. This finding was believed to be compatible with pulmonary thromboembolism; however, it didn't exclude a possible right pulmonary artery sarcoma. In this case, the only procedure that would rule out the presence of a right pulmonary artery sarcoma (without delays in diagnosis) with any reasonable certainty was surgery.

We decided to perform rigid bronchoscopy under general anaesthesia, followed by EBUS using an ultrasound bronchoscope (model BF-UC160F-OL8; Olympus, Tokyo, Japan). The use of EBUS allowed us to see, in real-time, a blood clot floating into the right main pulmonary artery not infiltrating the wall of blood vessel (see the video provided as online supplementary material). This finding confirmed the diagnostic hypothesis of pulmonary thromboembolism in a patient affected by antiphospholipid antibodies syndrome and avoided diagnostic surgery.

In conclusion, we believe that the indications for use of endobronchial ultrasound are manifold and are certainly greater than those so far recognised. This procedure should therefore be implemented and further developed in interventional pneumology.

\section{G.L. Casoni, C. Gurioli, M. Romagnoli and V. Poletti}

Thoracic Dept, G.B. Morgagni Hospital, Forlí, Italy.

\section{STATEMENT OF INTEREST}

None declared.

\section{SUPPLEMENTARY MATERIAL}

This article has supplementary material accessible from www.erj.ersjournals.com

\section{REFERENCES}

1 Herth F, Becker HD, LoCicero J 3rd, Ernst A., Endobronchial ultrasound in therapeutic bronchoscopy. Eur Respir J 2002; 20: 118-121.

2 Sheski FD, Mathur PN. Endobronchial ultrasound. Chest 2008; 133: 264-270.

\title{
Pneumonia in the context of severe sepsis: a significant diagnostic problem
}

\section{To the Editors:}

The Surviving Sepsis guidelines [1] highlighted a need to improve clinical standards for the management of severe sepsis and septic shock within the Nottingham University Hospitals NHS Trust (Nottingham, UK). We formed a multidirectorate task group to conduct a near real-time retrospective gap analysis of all adults with significant positive blood cultures of a pathogenic organism within the Queen's Medical Centre (Nottingham), between November 1, 2005 and March 31, 2006. The design, objectives and outcomes have been described previously [2]. Patients were included if they met the criteria for severe sepsis or septic shock, and were considered for full and active management. They were excluded if they were aged $<16$ yrs or were transferred from another hospital. During the study period, 229 suitable patients were identified and data were available from patient case notes for all subjects. Following exclusions, 46 subjects were analysed.

As part of the study, we recorded the diagnostic impression of the attending doctor at the start of the septic episode. The most common cause for the septic episode was recorded as pneumonia in $21(46 \%)$ subjects, urinary infection in $10(22 \%)$ subjects and biliary infection in five (11\%) subjects. Of the 21 subjects initially deemed to have pneumonia, only 15 had contemporary radiological changes supporting this diagnosis. 
TABLE 1 Blood culture flora of patients with severe sepsis or septic shock

\begin{tabular}{|c|c|c|c|c|}
\hline & $\begin{array}{l}\text { Initial impression of } \\
\text { pneumonia }\end{array}$ & $\begin{array}{c}\text { Consolidation on chest } \\
\text { radiograph }\end{array}$ & $\begin{array}{l}\text { Proven pneumonia in the } \\
\text { absence of alternative } \\
\text { sepsis source }\end{array}$ & $\begin{array}{l}\text { Expected organism } \\
\text { frequency }\end{array}$ \\
\hline Subjects $n$ & 21 & 17 & 9 & \\
\hline \multicolumn{5}{|l|}{ Organism } \\
\hline Streptococcus pneumoniae & $4(19)^{\circ}$ & $4(24)^{\circ}$ & $4(44)$ & 48 \\
\hline Legionella pneumophila & $2(10)$ & $2(12)$ & $2(22)$ & 3 \\
\hline Escherichia coli & $4(19)$ & $2(12)$ & $0(0)$ & $<3$ \\
\hline Haemophilus influenzae & $1(5)$ & $1(6)$ & $1(11)$ & 7 \\
\hline Enterobacter cloacae & $1(5)$ & $1(6)$ & $1(11)$ & $<3$ \\
\hline Klebsiella oxytoca & $2(10)$ & $1(6)$ & $0(0)$ & $<3$ \\
\hline Veillonella spp. & $1(5)$ & $0(0)$ & $0(0)$ & $<3$ \\
\hline
\end{tabular}

Furthermore, two patients whose sepsis was ascribed to other sources had consolidative changes on a chest radiograph. Review of the inpatient stays revealed that of these 17 severely septic patients with abnormal chest radiographs, only nine clearly had pneumonia in the absence of any other likely source of sepsis. The organisms cultured in blood samples obtained at the start of the septic episode are described in table 1.

These data indicate that pneumonia is being over-diagnosed as a cause for the systemic inflammatory response syndrome observed in severely septic patients. Of the 21 patients initially thought to have pneumonia, six (29\%) had no evidence of this on contemporary chest radiographs. These same patients tended to culture bacteria that would not classically cause pneumonia, such as Escherichia coli. Of these six patients, three $(50 \%)$ were started on antibiotics that were inappropriate for the organism eventually cultured, compared with three $(20 \%)$ out of the remaining 15 .

Streptococcus pneumoniae remained the most prevalent organism throughout all groups examined. However, the proportion of disease attributable to this organism was much higher in the group of patients who genuinely had pneumonia as the cause of their sepsis. On reflection, the distribution of organisms in this group is consistent with previously published local data [3], in contrast to the other groups examined.

In conclusion, there is an important proportion (29\%) of patients with severe sepsis or septic shock wrongly labelled with the diagnosis of pneumonia. This leads to inappropriate initial antibiotic use in the crucial first few days of the septic episode.

\section{T. Bewick, M. Simmonds, M. Chikhani, J. Meyer and W.S. Lim}

Nottingham University Hospitals NHS Trust, Nottingham, UK.

\section{STATEMENT OF INTEREST}

None declared.

\section{REFERENCES}

1 Dellinger RP, Carlet JM, Masur H, et al. Surviving Sepsis Campaign guidelines for management of severe sepsis and septic shock. Crit Care Med 2004; 32: 858-873.

2 Simmonds M, Hutchinson A, Chikhani M, et al. Surviving sepsis beyond intensive care: a retrospective cohort study into compliance with the international guidelines. JICS 2008; 9: $124-127$

3 Lim WS, Macfarlane JT, Boswell TC, et al. Study of community acquired pneumonia aetiology (SCAPA) in adults admitted to hospital: implications for management guidelines. Thorax 2001; 56: 296-301. 